\title{
A Francisella novicida $p d p A$ mutant exhibits limited intracellular replication and remains associated with the lysosomal marker LAMP-1
}

\section{Correspondence \\ Francis E. Nano \\ fnano@uvic.ca}

Received 24 October 2008

Revised 29 January 2009

Accepted 30 January 2009

\author{
Crystal L. Schmerk, ${ }^{1}$ Barry N. Duplantis, ${ }^{1}$ Perry L. Howard ${ }^{1,2}$ \\ and Francis E. Nano ${ }^{1}$
}

\author{
${ }^{1}$ Department of Biochemistry and Microbiology, University of Victoria, Victoria, BC, Canada \\ ${ }^{2}$ Department of Biology, University of Victoria, Victoria, BC, Canada
}

\section{INTRODUCTION}

Francisella tularensis is a facultative intracellular pathogen that is able to grow in a number of cell types, and is often found in infected tissues within cells of the monocytic cell lineage (Anthony et al., 1991; Bosio \& Dow, 2005; Conlan \& North, 1992; Ellis et al., 2002; Hall et al., 2007). In vitro studies have shown that $F$. tularensis subverts the normal endocytic pathway of host macrophages and grows rapidly within these cells (Santic et al., 2005a). Approximately 20 min after macrophage engulfment, the Francisellacontaining phagosome acquires the early endosomal markers EEA-1 and Rab5 (Clemens et al., 2004). The phagosome subsequently gains late endosomal markers such as Rab7, CD63, LAMP-1 and LAMP-2 (Clemens et al., 2004; Santic et al., 2005a). This late endosome-like compartment acquires the proton vacuolar ATPase pump and becomes transiently acidified but does not associate with lysosomal markers, such as cathepsin D (Clemens et al., 2004; Santic et al., 2005a, 2008). Francisella is thus able to prevent phagosomal-lysosomal fusion, and within $4 \mathrm{~h}$ of uptake actively breaks down the phagosomal membrane in order to escape into the host-cell cytosol and replicate. The infective process eventually leads to

Abbreviations: BMDM, bone-marrow-derived macrophages; FPI, Francisella pathogenicity island; T6SS, type VI secretion system.

Three supplementary tables are available with the online version of this paper. host-cell death, whereby the bacteria are freed to infect neighbouring cells.

All Francisella species contain at least one copy of a gene cluster known as the Francisella pathogenicity island (FPI). A number of genes, particularly within the FPI, have been shown necessary for phagosomal escape and intracellular replication; however, most of these studies have not investigated the nature of the gene products nor provided any evidence to support a mechanism of action of the gene products (Bonquist et al., 2008; de Bruin et al., 2007; Nano et al., 2004; Santic et al., 2005b, 2008). Studies of mutants of Francisella novicida that have knockouts of genes encoding phosphatases suggest that one or more of them play a role in phagosome membrane degradation. Since the acid phosphatase, AcpA, also has lipase activity, it may play an important role in degrading the membrane (Mohapatra et al., 2007a, 2008; Reilly et al., 1996).

Microarray analysis has recently been employed in a variety of ways to study Francisella infection. The majority of these studies have used microarray technology to profile the transcriptional response of Francisella-infected immune cells (Andersson et al., 2006a, b; Butchar et al., 2008; Paranavitana et al., 2008). Some have used the microarraybased studies to identify virulence genes (Weiss et al., 2007) or study the control of virulence gene expression by regulators such as $p m r A, m g l A$ and sspA (Charity et al., 2007; Mohapatra et al., 2007b; Sammons-Jackson et al., 
2008). Microarray analysis has not hitherto been used to profile the change in host-cell responses to Francisella strains with mutations in virulence genes.

The $p d p A$ gene is one of the largest in the FPI and is located at the beginning of a putative operon containing the $p d p B, \operatorname{vgr} G$ and $\operatorname{dot} U$ genes. A previously described gene replacement mutant in the $p d p A$ gene of $F$. novicida exhibited impaired intracellular replication and avirulence in mice; however, the substitution of the erythromycin resistance $\left(\mathrm{Em}^{\mathrm{R}}\right)$ cassette for $p d p A$ has since been shown by us (unpublished data) to have polarity effects on genes downstream of $p d p A$. Because many of these downstream genes affect intracellular growth, it is possible that the altered intracellular growth phenotype of the $\Delta p d p A:: \mathrm{Em}^{\mathrm{R}}$ mutant was due to the suppressed expression of multiple FPI-encoded proteins. In a companion paper (Schmerk et al., 2009) we describe a non-polar, $F$. novicida, $p d p A$ deletion mutant and show that it is highly attenuated for virulence. In this paper we examine the intracellular growth phenotype of the $\triangle p d p A$ mutant, and the effect of the deletion of $p d p A$ on macrophage gene expression response to $F$. novicida infection.

\section{METHODS}

Bacterial strains and growth conditions. The bacterial strains used in this study are listed in Table 1. F. novicida strains were grown using trypticase soy agar or broth supplemented with $0.1 \%(\mathrm{w} / \mathrm{v})$ cysteine (TSAC, TSBC). When necessary, $15 \mu \mathrm{g}$ kanamycin $\mathrm{ml}^{-1}$ was added to the growth medium.

Intracellular growth assays. Bone marrow cells were isolated from the femurs of male BALB/c mice and seeded in 96-well cell culture plates at a density of $3 \times 10^{5}$ cells per well. Cells were incubated for 1 week in complete Dulbecco's Modified Eagle Medium (cDMEM) supplemented with $10 \%$ fetal bovine serum, $2 \mathrm{mM}$ L-glutamine, $1 \%$ MEM nonessential amino acids, $10 \mathrm{mM}$ HEPES buffer solution and $10 \%$ conditioned L929 cell supernatant. After 7 days the macrophages were infected with $F$. novicida strains at an m.o.i. of $50: 1$ (bacteria:macrophage). The monolayers were incubated at $37^{\circ} \mathrm{C}$, $5 \% \mathrm{CO}_{2}$ for $1 \mathrm{~h}$ to allow uptake of the bacteria and then washed five times using sterile PBS. Infected macrophages were lysed with $0.1 \%$ $(\mathrm{w} / \mathrm{v})$ deoxycholic acid at 0,24 and $48 \mathrm{~h}$ post-infection. Lysates were serially diluted in PBS containing $0.1 \%(\mathrm{w} / \mathrm{v})$ gelatin and plated on TSAC for enumeration.

Experiments using the J774A.1 murine macrophage cell line were carried out in a manner similar to those used for bone marrow- derived macrophages (BMDM). Cells were seeded in 96-well cell culture plates at a density of $5 \times 10^{4}$ cells per well and allowed to adhere overnight. F. novicida strains were added to the wells at an m.o.i. of 50:1 and the infection was carried out as described above. Infected macrophages were lysed with $0.1 \%$ deoxycholic acid at 0,24 and $48 \mathrm{~h}$ post-infection.

Real-time PCR assays. J774A.1 murine macrophage infections were performed as described above, except that infections were performed in $25 \mathrm{~cm}^{2}$ tissue culture flasks. mRNA was isolated from the macrophage cell line $12 \mathrm{~h}$ after infection using PureLink Micro-toMidi total RNA purification system (Invitrogen) according to the manufacturer's protocols. Quantitative real-time (qRT-PCR) data were generated using the $\mathrm{RT}^{2}$ Profiler PCR array mouse Signal Transduction PathwayFinder (SuperArray Bioscience). qRT-PCR was performed with $1.5 \mu \mathrm{g}$ total RNA according to the manufacturer's protocol using the Stratagene MX4000 thermocycler.

Immunofluorescence and LAMP-1 association. J774A.1 macrophages were seeded on $22 \mathrm{~mm}$ glass coverslips in 6-well tissue culture plates at a density of $1 \times 10^{6}$ cells per well and allowed to adhere overnight. Cells were chilled on ice for $5 \mathrm{~min}$ and bacteria were added to each well at an m.o.i. of $500: 1$, after which the cells were chilled for an additional $10 \mathrm{~min}$. Cells were immediately warmed in a $37{ }^{\circ} \mathrm{C}$ water bath for 3 min to synchronize bacterial uptake and then incubated at $37{ }^{\circ} \mathrm{C}, 5 \% \mathrm{CO}_{2}$ for an additional $20 \mathrm{~min}$. Wells were washed five times in PBS and fresh medium was added. At the appropriate time points the coverslips were removed and rinsed in PBS. The coverslips were then fixed in $2 \%(\mathrm{w} / \mathrm{v})$ paraformaldehyde, $1 \%(\mathrm{w} / \mathrm{v})$ sucrose, PBS for $20 \mathrm{~min}$ at room temperature followed by immersion in ice-cold methanol for 10 min at $-20{ }^{\circ} \mathrm{C}$. Coverslips were blocked in PBS containing $5 \%$ lamb serum for $30 \mathrm{~min}$. Rabbit polyclonal anti- $F$. novicida (Nano, 1988) and rat monoclonal anti-LAMP-1 (DSHB, University of Iowa) antibodies were incubated with the coverslips overnight at $4{ }^{\circ} \mathrm{C}$ at a dilution of $1: 1000$. Coverslips were then washed three times with PBS and incubated for $2 \mathrm{~h}$ with goat anti-rabbit Alexafluor568 and goat anti-rat Alexafluor488 secondary antibodies (Molecular Probes) at a dilution of $1: 1500$. The coverslips were incubated for $5 \mathrm{~min}$ with Hoescht 33258 (Molecular Probes) to label the DNA. Cells were imaged with a Leica DMIREZ inverted fluorescent microscope using a $100 \times$ oil immersion lens. Using Openlab 5.1 software, multiple-channel $Z$ stacks were captured and deconvoluted in order to score macrophage LAMP-1 association with the bacterial strains.

Graphing and statistics. The Prism GraphPad v4.03 software was used to generate graphs and to calculate the appropriate statistical values, including standard deviation, standard error of the mean and $P$-values. For $P$-values the Student $t$-test or two-way ANOVA were used where appropriate.

Table 1. Bacterial strains used in this study

\begin{tabular}{|c|c|c|}
\hline Strain & Characteristics & Reference \\
\hline U112 & Wild-type F. novicida & ATCC 15482 \\
\hline $\mathrm{JLO}$ & $\begin{array}{l}\text { U112 with deletion in FTN_1390, where SKX vector inserts; identical } \\
\text { growth and virulence with respect to U112 }\end{array}$ & Ludu et al. (2008b) \\
\hline GB2 & U112 with point mutation in global virulence regulator, $\mathrm{mglA}$ & Baron \& Nano (1998) \\
\hline$\Delta p d p A$ & JLO with a deletion of $p d p A$ & This study \\
\hline$\Delta p d p A / S K X:: p d p A$ & $\Delta p d p A$ complemented with the integrating $\mathrm{pJL}-\mathrm{SKX}:: p d p A$ construct & This study \\
\hline$\Delta i g l C$ & JLO with a deletion of $i g l C$ & de Bruin et al. (2007) \\
\hline
\end{tabular}




\section{RESULTS}

\section{Intracellular growth of $p d p A$ mutants}

Our previous work had shown that a gene replacement mutation in $p d p A$ resulted in a strain defective for intramacrophage growth (Nano et al., 2004). We have since learned that this and other replacement mutations generated polarity effects; hence we examined the phenotype of the newly created $\triangle p d p A$ strain (Schmerk et al., 2009), which has a non-polar deletion mutation. Infection of the $\triangle p d p A$ mutant in mouse BMDM resulted in limited growth that showed an initial increase in $F$. novicida numbers followed by a decline in numbers (Fig. 1a). Macrophages infected with the $\Delta p d p A$ mutant appeared healthy throughout the infection, displaying no signs of cytotoxicity or cell-rounding. Genetic complementation, but not a mock complementation (data not shown) with
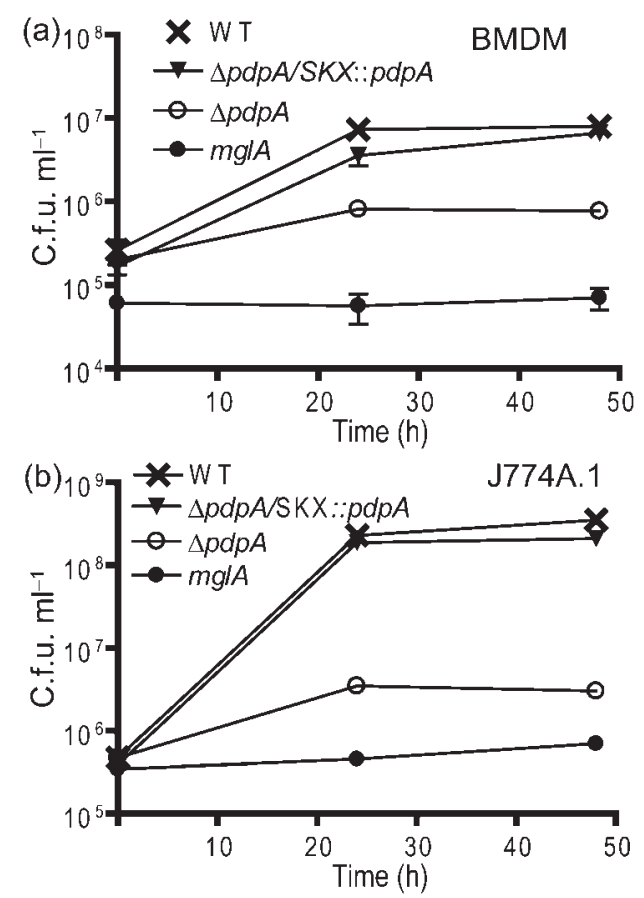

Fig. 1. Intracellular growth of $\Delta p d p A$ mutants. The $\Delta p d p A$ mutant was able to replicate in BMDM (a) and J774A.1 cells (b) within the first $24 \mathrm{~h}$ of infection. Bacterial numbers decreased after $48 \mathrm{~h}$ and macrophages exhibited no signs of cytotoxicity. Complementation of the $\triangle p d p A$ deletion restored the wild-type (WT) growth phenotype. The $\Delta m g / A$ mutant was included as a control as it is unable to replicate within macrophages. All data points in both panels are representative of three replicates and each experiment was performed in triplicate. Two-way ANOVA was used to calculate the significance of the differences in the growth curves between pairs of strains. For BMDM: WT vs $\triangle p d p A, P<0.00$; WT vs $\Delta p d p A / S K X:: p d p A, P=0.0261$; and for $\Delta p d p A$ vs $\Delta p d p A /$ SKX:: $p d p A, P=0.0028$. For J774A.1 macrophages: WT vs $\Delta p d p A, P=<0.001$; WT vs $\Delta p d p A / S K X:: p d p A, P=0.006$; and for $\triangle p d p A$ vs $\triangle p d p A / S K X:: p d p A, P=<0.001$. the pJL-SKX vector, restored nearly complete growth in the macrophages (Fig. 1a). Very similar results were observed when J774A.1 macrophages were infected with the $\Delta p d p A$ mutant and its genetic complement (Fig. 1b). While statistical analysis showed that the growth of the $\Delta p d p A$ mutant is clearly different from both the wild-type strain and from the complemented strain, this analysis also showed that the growth of the wild-type and complemented strains was different, indicating that the wild-type phenotype was not fully restored by genetic complementation. The influence of an exogenous promoter in the pJLSKX:: $p d p A$ vector may account for these differences.

\section{LAMP-1 association of the pdpA mutant}

There is now a substantial body of literature that documents the escape of Francisella from LAMP-1-laden phagosomal vesicles, and the failure of some FPI mutants to escape at wild-type levels. In order to evaluate the intracellular trafficking of the $\triangle p d p A$ mutant, we infected mouse macrophage-like cell line J774A.1 cells, and examined the co-localization of LAMP-1 with wild-type F. novicida, the $\triangle p d p A$ mutant, and a $\Delta i g l C$ mutant. $F$. tularensis and F. novicida mutants in iglC have been shown by others to be defective for phagosome escape (Bonquist et al., 2008; Lindgren et al., 2004; Santic et al., 2005b). Our experimental results using deconvoluting fluorescence microscopy showed a clear separation of wild-type $F$. novicida and LAMP-1 localization at $12 \mathrm{~h}$ post-infection (Fig. 2a-c). In contrast, the $\Delta p d p A$ mutant (Fig. $2 \mathrm{~d}-\mathrm{f}$ ) and the $\triangle i g l C$ mutant (Fig. $2 \mathrm{~g}-\mathrm{i}$ ) showed close association of $F$. novicida and LAMP-1 localization. A close examination of the micrographs revealed that individual cells of $\Delta p d p A$ (Fig. $2 \mathrm{~d}-\mathrm{f}$ ) and $\Delta i g l C$ (Fig. $2 \mathrm{~g}-\mathrm{i}$ ) were surrounded by a LAMP-1-laden structure that took the shape of the bacterial cells. However, this was not seen in the images of wild-type F. novicida (Fig. 2a-c). Genetic complementation with pJL-SKX : :pdpA restored LAMP-1 association to wild-type levels (data not shown). The clear association of $\triangle p d p A$ cells within a LAMP-1-loaded structure continued even after $19 \mathrm{~h}$ infection (Fig. 3a-c). A quantitative analysis of $F$. novicida-LAMP-1 association showed that less than $10 \%$ of the wild-type $F$. novicida was LAMP-1 associated 12 h post-infection while $92 \%$ of $\Delta p d p A$ and $74 \%$ of the $\triangle i g l C$ strain were LAMP-1 associated (Fig. 4).

\section{Effect of the deletion of pdpA on host-cell mRNA responses}

As an approach to discern a possible role of PpdA in hostcell processes, we compared the effect of infection of macrophages with wild-type and the $\Delta p d p A$ deletion strain on host-cell mRNA levels of selected signalling pathways. A summary from a representative set of experiments of the mRNA levels that were affected is shown in Table 2 and the complete dataset is presented in Supplementary Tables S1S3 (available with the online version of this paper). Each of the columns showing the fold changes represents the 

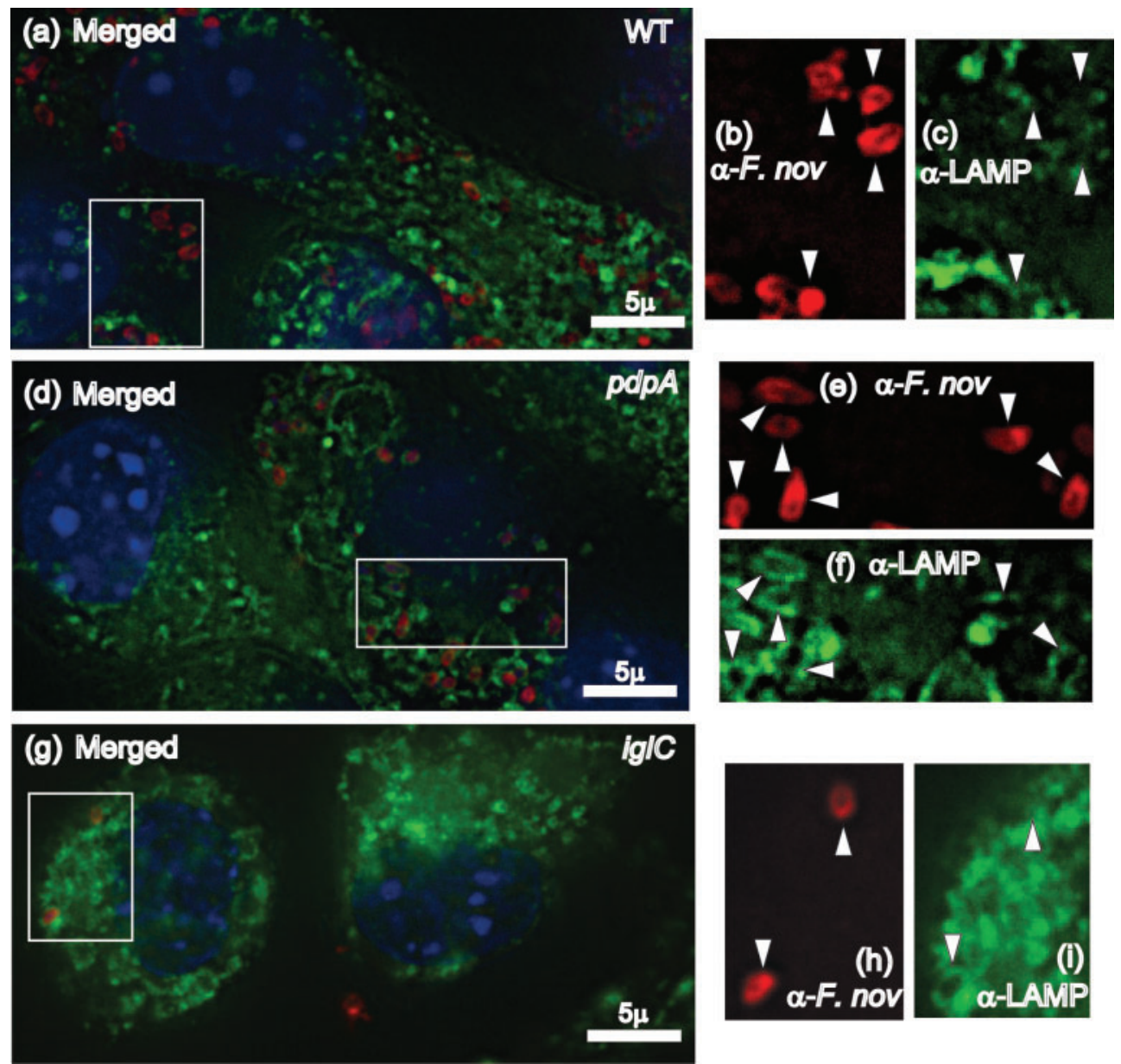

Fig. 2. The $\triangle p d p A$ mutant replicates in macrophages but remains LAMP-1 associated. J774A.1 macrophages adhered to glass coverslips were infected with $F$. novicida $\mathrm{U} 112(\mathrm{a}-\mathrm{c}), \Delta p d p A(\mathrm{~d}-\mathrm{f})$, or $\Delta i g / C(\mathrm{~g}-\mathrm{i})$ at an m.o.i. of $500: 1$. The colours in merged panels a, $d$ and g correspond to Hoechst DNA (blue), anti-LAMP-1 (green), and anti-F. novicida (red). Panels b, e and $\mathrm{h}$ represent staining with anti- $F$. novicida antibody, and panels $c, f$ and $i$ represent staining with anti-LAMP-1. The arrowheads indicate areas of LAMP-1 staining that correspond to bacterial location.

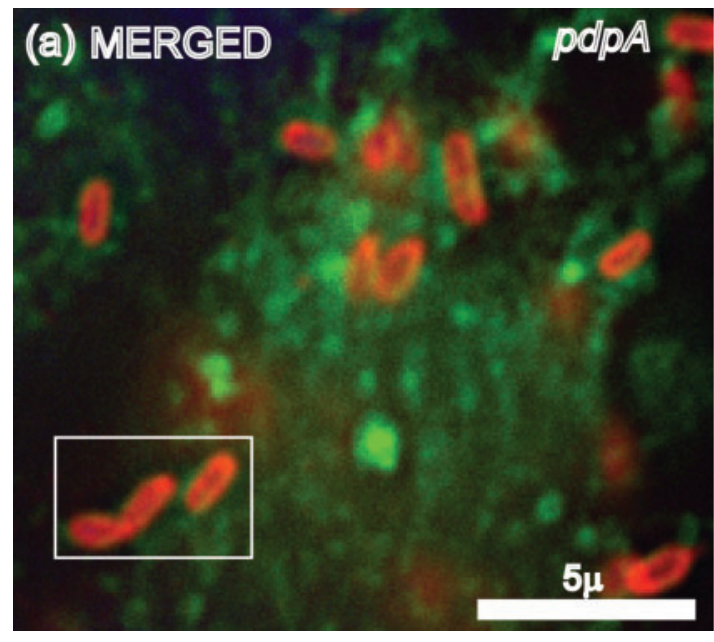

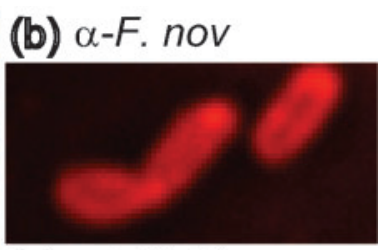

(c) $\alpha$-LAMP

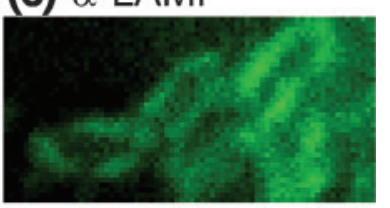

Fig. 3. The $\triangle p d p A$ mutant remains LAMP-1 associated late in infection. The association of $\triangle p d p A$ with LAMP-1 continues after $19 \mathrm{~h}$ infection (a). This localization is clearly seen in the corresponding enlarged images of the boxed region stained with only anti- $F$. novicida (b) or anti-LAMP-1 (c). 


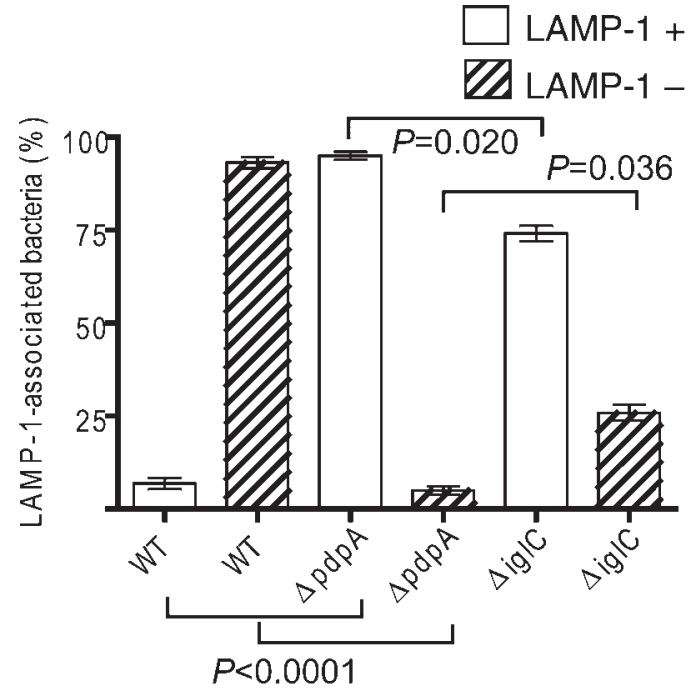

Fig. 4. Association of $F$. novicida mutants with LAMP-1. Bacterial association with LAMP-1 was scored at $12 \mathrm{~h}$ post-infection by counting a minimum of $100 \mathrm{~F}$. novicida cells for each strain. The Student's $t$-test was used to examine the significance of the differences between strains of the LAMP-1 positive or negative $F$. novicida cells and some of these $P$-values are shown on the graph. The $P$-values for wild-type (WT) F. novicida vs the $\Delta i g / C$ strain are (LAMP-1 positive), 0.0001; and (LAMP-1 negative), 0.0024. The experiment was performed in triplicate.

results of separate experiments in which the mRNA levels were measured from sets of macrophage cultures subjected to two different infections or an infection and a mock infection. In addition, all experimental runs were duplicated. When the mRNA levels following infection with the $\triangle p d p A$ strain are compared to those following infection with the wild-type strain there are 28 mRNA species that are elevated and four that are depressed (Table 2, column 3 ) at least twofold. Importantly, the experimental fold differences seen for infections with the $\Delta p d p A$ vs the wildtype strain (column 3) are reflected by a comparison between the results of a wild-type infection vs uninfected macrophages (column 5) and the results of the mRNA levels measured in $\triangle p d p A$ infections vs uninfected macrophages (column 7). Of the 32 RNAs shown in Table 2, 13 encode ligands, five encode receptors, six encode pathway components and eight encode effector molecules. Of the ten most strongly upregulated messages, eight are transcripts from ligand-encoding genes.

\section{DISCUSSION}

Like many intracellular pathogens, the ability of $F$. tularensis to interfere with the endocytic pathway of its host cell is vital in the pathogen's ability to cause disease. Although it is clear that infecting bacteria are able to break down the phagocytic membrane prior to lysosomal fusion, it is not clear what virulence factors are responsible for this process. It is apparent that many of the FPI-encoded proteins are required for Francisella intracellular growth and virulence, although some of the pathogenicity island proteins are required only for the latter (Ludu et al., 2008a). There is also substantial bioinformatic and biochemical evidence that the FPI encodes proteins that make up a type VI secretion apparatus or a macromolecular structure related to the type VI secretion system (T6SS) (Bingle et al., 2008; de Bruin et al., 2007; Ludu et al., 2008a). Although we and others have adopted the hypothesis that an FPI-encoded secretion system is responsible for transport of virulence factors that modulate host-cell functions, there is, at present, little evidence for this model, and no experimental results that precisely define the role of any FPI-encoded protein.

This study represents the first detailed analysis of a nonpolar $p d p A$ deletion mutant and the role of PdpA in intracellular growth. Although the $\triangle p d p A$ mutant is able to replicate, albeit minimally, in both BMDM and J774A.1 macrophages, this replication ceases after $24 \mathrm{~h}$ and bacterial burdens begin to decrease. This growth phenotype differs from that of a previously studied polar allelic replacement mutant that was completely impaired in its ability to replicate within macrophages (Nano et al., 2004). Recent work by Chong et al. (2008) reveals that polarity effects are also observed in the $\Delta i g l C:$ :Em mutant, with levels of IglD expression being drastically reduced in this strain. These authors suggest that such observations require the re-evaluation of conclusions drawn from previous studies using the $\Delta i g l C:$ :Em mutant. This agrees with our findings regarding the $\triangle p d p A::$ Em mutant and emphasizes the importance of creating non-polar mutants for analysis, especially in the FPI, where the full expression of several transcriptionally linked genes appears to be required for intracellular growth. Statistical analysis indicated that genetic complementation of the $p d p A$ deletion did not fully restore intracellular growth to wildtype levels. The incomplete complementation may have resulted from the recombinant $p d p A$ gene being located at an ectopic location within the chromosome, leading to subtle alterations in $p d p A$ expression.

Other researchers have shown that mutation of some FPI genes leads to Francisella strains that fail to escape the phagosome (Bonquist et al., 2008; Lindgren et al., 2004; Santic et al., 2005b). Like this work, these studies are attempts to surmise the function of different FPI-encoded proteins by examining the intramacrophage phenotype of Francisella strains with lesions in FPI genes. The deletion mutant of $p d p A$ made in this work behaves similarly to mutants in $i g l C$ and $i g l D$, in that all the mutants are impaired in their intracellular replication and have an increased association with the LAMP-1 lysosomal marker at time points when wild-type Francisella has escaped the phagosome (Bonquist et al., 2008). Since the FPI appears to encode a T6SS, the current challenge is to decipher which FPI genes are needed for secretion and which, if any, encode secreted effector proteins that interact with host- 
Table 2. Changes in mRNA levels of $J 744$ macrophage-like cells following infection with $F$. novicida strains

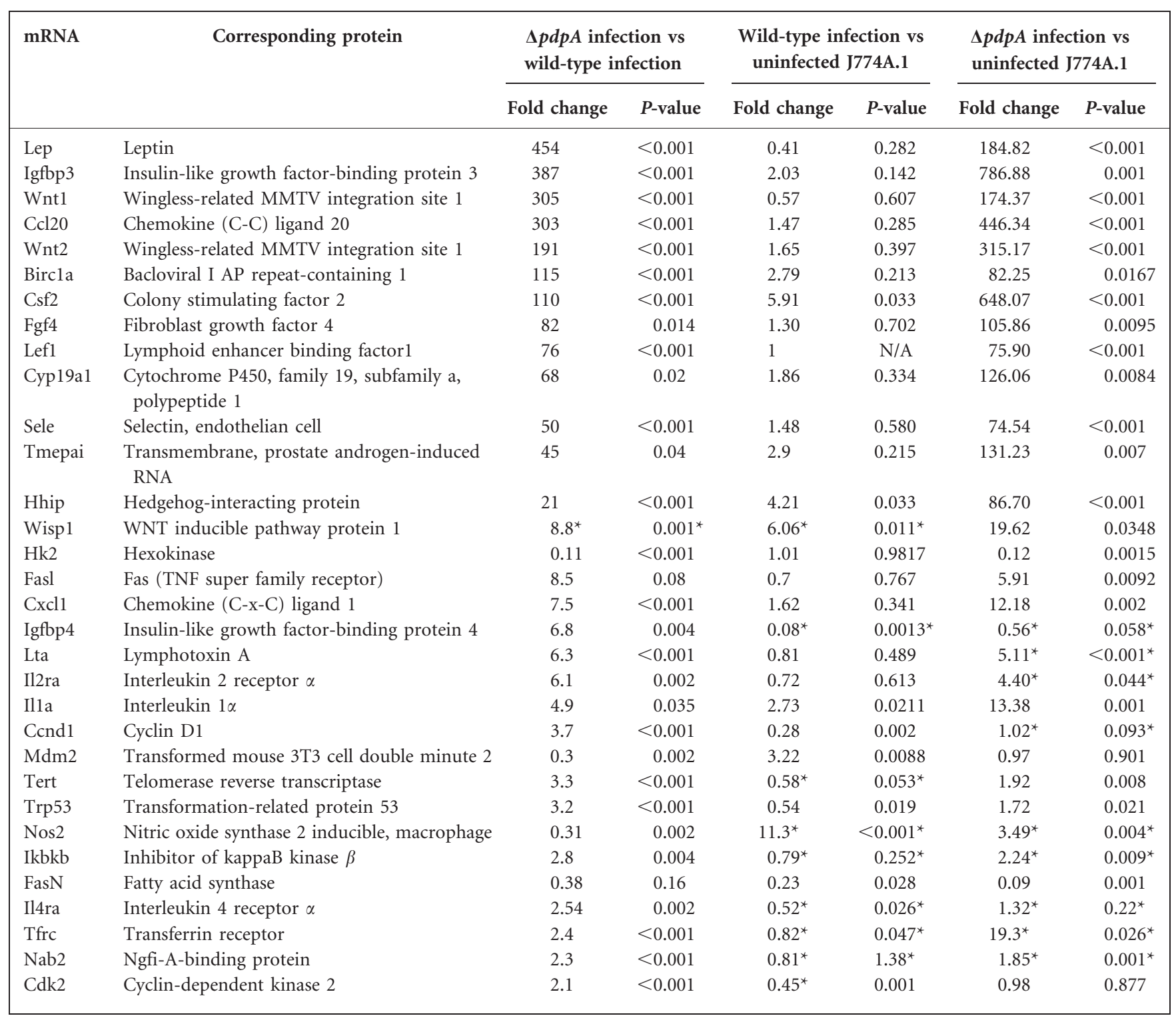

${ }^{\star}$ Indicates that a value represents the mean of four samples rather than five in an experimental run.

cell components. We do not believe that PdpA is a structural component of the T6SS because the protein does not seem to play a part in the secretion of IglC (Ludu et al., 2008a, and data not shown), and bioinformatic analysis fails to show any similarity of PdpA with known T6SS components in other bacteria.

Without knowing the nature of $\mathrm{PdpA}$, and whether its mode of action has a direct or indirect effect on host-cell function, it is difficult to attribute a biological response directly to its expression. Nevertheless, our comparison of host-cell mRNA responses to infection by wild-type $F$. novicida and to the $\Delta p d p A$ mutant may contribute to the development of hypotheses about the role of PdpA and its interaction with host-cell components. Our examination of the levels of selected host-cell transcripts shows that the absence of PdpA in F. novicida results in significantly different host-cell responses to infection compared to an infection with wild-type $F$. novicida. That the absence of $\mathrm{PdpA}$ results in higher levels of mRNA for genes encoding ligands suggests that one of PdpA's functions is to suppress macrophage ability to signal via growth factors, cytokines and adhesion ligands. Our inference from these limited data is that PdpA plays a role in suppressing the infected macrophage's ability to recruit and stimulate other immune cells. However, these results can only be considered as clues to the role of PdpA in the intracellular parasitism by F. novicida, and considerably more work is needed to define the role and mode of action of PdpA. 


\section{ACKNOWLEDGEMENTS}

This work was supported by grants 5R01 AI056212-02 from the National Institute of Allergy and Infectious Diseases and MOP 89812 from the Canadian Institutes of Health Research to F.E. N.

\section{REFERENCES}

Andersson, H., Hartmanova, B., Kuolee, R., Ryden, P., Conlan, W., Chen, W. \& Sjostedt, A. (2006a). Transcriptional profiling of host responses in mouse lungs following aerosol infection with type A Francisella tularensis. J Med Microbiol 55, 263-271.

Andersson, H., Hartmanova, B., Ryden, P., Noppa, L., Naslund, L. \& Sjostedt, A. (2006b). A microarray analysis of the murine macrophage response to infection with Francisella tularensis LVS. J Med Microbiol 55, 1023-1033.

Anthony, L. D., Burke, R. D. \& Nano, F. E. (1991). Growth of Francisella spp. in rodent macrophages. Infect Immun 59, 3291-3296.

Baron, G. S. \& Nano, F. E. (1998). MglA and MglB are required for the intramacrophage growth of Francisella novicida. Mol Microbiol 29, 247-259.

Bingle, L. E., Bailey, C. M. \& Pallen, M. J. (2008). Type VI secretion: a beginner's guide. Curr Opin Microbiol 11, 3-8.

Bonquist, L., Lindgren, H., Golovliov, I., Guina, T. \& Sjostedt, A. (2008). The MglA and Igl proteins contribute to the modulation of Francisella tularensis LVS-containing phagosomes in murine macrophages. Infect Immun 76, 3502-3510.

Bosio, C. M. \& Dow, S. W. (2005). Francisella tularensis induces aberrant activation of pulmonary dendritic cells. J Immunol $\mathbf{1 7 5}$, 6792-6801.

Butchar, J. P., Cremer, T. J., Clay, C. D., Gavrilin, M. A., Wewers, M. D., Marsh, C. B., Schlesinger, L. S. \& Tridandapani, S. (2008). Microarray analysis of human monocytes infected with Francisella tularensis identifies new targets of host response subversion. PLoS One 3, e2924.

Charity, J. C., Costante-Hamm, M. M., Balon, E. L., Boyd, D. H., Rubin, E. J. \& Dove, S. L. (2007). Twin RNA polymerase-associated proteins control virulence gene expression in Francisella tularensis. PLoS Pathog 3, e84.

Chong, A., Wehrly, T. D., Nair, V., Fischer, E. R., Barker, J. R., Klose, K. E. \& Celli, J. (2008). The early phagosomal stage of Francisella tularensis determines optimal phagosomal escape and Francisella pathogenicity island protein expression. Infect Immun 76, 5488-5499.

Clemens, D. L., Lee, B. Y. \& Horwitz, M. A. (2004). Virulent and avirulent strains of Francisella tularensis prevent acidification and maturation of their phagosomes and escape into the cytoplasm in human macrophages. Infect Immun 72, 3204-3217.

Conlan, J. W. \& North, R. J. (1992). Early pathogenesis of infection in the liver with the facultative intracellular bacteria Listeria monocytogenes, Francisella tularensis, and Salmonella typhimurium involves lysis of infected hepatocytes by leukocytes. Infect Immun 60, 5164-5171.

de Bruin, O. M., Ludu, J. S. \& Nano, F. E. (2007). The Francisella pathogenicity island protein IglA localizes to the bacterial cytoplasm and is needed for intracellular growth. BMC Microbiol 7, 1.

Ellis, J., Oyston, P. C., Green, M. \& Titball, R. W. (2002). Tularemia. Clin Microbiol Rev 15, 631-646.

Hall, J. D., Craven, R. R., Fuller, J. R., Pickles, R. J. \& Kawula, T. H. (2007) Francisella tularensis replicates within alveolar type II epithelial cells in vitro and in vivo following inhalation. Infect Immun 75, 1034-1039.

Lindgren, H., Golovliov, I., Baranov, V., Ernst, R. K., Telepnev, M. \& Sjostedt, A. (2004). Factors affecting the escape of Francisella tularensis from the phagolysosome. J Med Microbiol 53, 953-958.
Ludu, J. S., de Bruin, O. M., Duplantis, B. N., Schmerk, C. L., Chou, A. Y., Elkins, K. L. \& Nano, F. E. (2008a). The Francisella pathogenicity island protein $\mathrm{PdpD}$ is required for full virulence and associates with homologues of the type VI secretion system. J Bacteriol 190, 4584-4595.

Ludu, J. S., Nix, E. B., Duplantis, B. N., de Bruin, O. M., Gallagher, L. A., Hawley, L. M. \& Nano, F. E. (2008b). Genetic elements for selection, deletion mutagenesis and complementation in Francisella spp. FEMS Microbiol Lett 278, 86-93.

Mohapatra, N. P., Balagopal, A., Soni, S., Schlesinger, L. S. \& Gunn, J. S. (2007a). AcpA is a Francisella acid phosphatase that affects intramacrophage survival and virulence. Infect Immun 75, 390-396.

Mohapatra, N. P., Soni, S., Bell, B. L., Warren, R., Ernst, R. K., Muszynski, A., Carlson, R. W. \& Gunn, J. S. (2007b). Identification of an orphan response regulator required for the virulence of Francisella spp. and transcription of pathogenicity island genes. Infect Immun 75, 3305-3314.

Mohapatra, N. P., Soni, S., Reilly, T. J., Liu, J., Klose, K. E. \& Gunn, J. S. (2008). The combined deletion of four Francisella acid phosphatases attenuates virulence and macrophage vacuolar escape. Infect Immun 76, 3690-3699.

Nano, F. E. (1988). Identification of a heat-modifiable protein of Francisella tularensis and molecular cloning of the encoding gene. Microb Pathog 5, 109-119.

Nano, F. E., Zhang, N., Cowley, S. C., Klose, K. E., Cheung, K. K., Roberts, M. J., Ludu, J. S., Letendre, G. W., Meierovics, A. I. \& other authors (2004). A Francisella tularensis pathogenicity island required for intramacrophage growth. J Bacteriol 186, 6430-6436.

Paranavitana, C., Pittman, P. R., Velauthapillai, M., Zelazowska, E. \& Dasilva, L. (2008). Transcriptional profiling of Francisella tularensis infected peripheral blood mononuclear cells: a predictive tool for tularemia. FEMS Immunol Med Microbiol 54, 92-103.

Reilly, T. J., Baron, G. S., Nano, F. E. \& Kuhlenschmidt, M. S. (1996). Characterization and sequencing of a respiratory burst-inhibiting acid phosphatase from Francisella tularensis. J Biol Chem 271, 10973-10983.

Sammons-Jackson, W. L., McClelland, K., Manch-Citron, J. N., Metzger, D. W., Bakshi, C. S., Garcia, E., Rasley, A. \& Anderson, B. E. (2008). Generation and characterization of an attenuated mutant in a response regulator gene of Francisella tularensis live vaccine strain (LVS). DNA Cell Biol 27, 387-403.

Santic, M., Molmeret, M. \& Abu Kwaik, Y. (2005a). Modulation of biogenesis of the Francisella tularensis subsp. novicida-containing phagosome in quiescent human macrophages and its maturation into a phagolysosome upon activation by IFN-gamma. Cell Microbiol 7, 957-967.

Santic, M., Molmeret, M., Klose, K. E., Jones, S. \& Kwaik, Y. A. (2005b). The Francisella tularensis pathogenicity island protein IglC and its regulator $\mathrm{MglA}$ are essential for modulating phagosome biogenesis and subsequent bacterial escape into the cytoplasm. Cell Microbiol 7, 969-979.

Santic, M., Asare, R., Skrobonja, I., Jones, S. \& Abu Kwaik, Y. (2008). Acquisition of the vacuolar ATPase proton pump and phagosome acidification are essential for escape of Francisella tularensis into the macrophage cytosol. Infect Immun 76, 2671-2677.

Schmerk, C. L., Duplantis, B. N., Wang, D., Burke, R. D., Chou, A. Y., Elkins, K. L., Ludu, J. S. \& Nano, F. E. (2009). Characterization of the pathogenicity island protein PdpA and its role in the virulence of Francisella novicida. Microbiology 155, 1489-1497.

Weiss, D. S., Brotcke, A., Henry, T., Margolis, J. J., Chan, K. \& Monack, D. M. (2007). In vivo negative selection screen identifies genes required for Francisella virulence. Proc Natl Acad Sci U S A 104, 6037-6042.

Edited by: T. P. Hatch 